\title{
Ein neuer Fragebogen zur Beurteilung der Weiterbildungsstätten - was soll das?
}

\author{
Dr. med. Max Giger, Leiter Ressort Medical Education FMH \\ PD Dr. phil. Michael Siegrist, Sozialforschungsstelle der Universität Zürich
}

Seit sechs Jahren erfolgt im Spätsommer eine Umfrage bei den Assistenzärztinnen und Assistenzärzten zur Qualität der Weiterbildungsstätten. Die Antworten erlauben jeweils eine globale Beurteilung der Weiterbildungsstätten, sind aber wenig geeignet, die Ursachen beanstandeter Mängel zu erkennen und mit gezielten Massnahmen zu beheben. In zwei Fällen wurde aufgrund der Resultate der Umfrage eine Visitation der Weiterbildungsstätte durch die Fachgesellschaft durchgeführt. In beiden Fällen konnte der Prozess der Weiterbildung verbessert werden.
Prätest gesammelten Erfahrungen und Kritiken wurden bei der Überarbeitung des Fragebogens berücksichtigt. Der neue Fragebogen wird in der zweiten Hälfte August den Leiterinnen und Leitern der Weiterbildungsstätten zur Verteilung an die Assistenzärztinnen und Assistenzärzte zugeschickt.

\section{Durchführung und Anonymität}

Der neue Fragebogen lehnt sich formal an die bisherigen an, ist etwas ausführlicher, jedoch innerhalb weniger als einer Viertelstunde zu beantworten. Er ist gegliedert in Fragen zur Kultur der Weiterbildung, zur Durchführung der Weiterbildung, zur Person der Assistenzärztinnen und Assistenzärzte und zum Studium. Diese letzten Fragen werden einzig in diesem Jahr gestellt. Sie sollen u.a. Aufschluss geben über die Praxisrelevanz des Studiums. Die Fragen zur Person dienen zur Interpretation der Beantwortung der anderen Fragen. Alle Daten bleiben bei der Sozialforschungsstelle. Sowohl die FMH als auch die Leiterinnen und Leiter der Weiterbildungsstätten erhalten eine Auswertung anonymisierter und zusammengefasster Daten.

Liegen von einer Weiterbildungsstätte mindestens vier ausgefüllte Fragebogen vor, so werden die Leiterinnen und Leiter der Weiterbildungsstätte eine Rückmeldung mit den Durchschnittswerten erhalten. Bei weniger als vier Fragebogen ist die Anonymität nur noch beschränkt gewährleistet. Deshalb können die Assistenzärztinnen und Assistenzärzte neu darüber entscheiden, ob eine Rückmeldung auch dann erfolgen darf, wenn weniger als vier ausgefüllte Fragebogen vorliegen. Bei weniger als vier Fragebogen werden nur jene für eine Rückmeldung an die Weiterbildungsstätte benutzt, bei denen das Einverständnis für eine Rückmeldung gegeben wurde. Dadurch werden auch bei weniger als vier Weiterzubildenden Rückmeldungen an die Leiterinnen und Leiter der Weiterbildungsstätten möglich. 


\section{Konsequenzen der Umfrage}

Die Resultate dieser Umfrage nützen den Weiterbildungsstätten, den Assistenzärztinnen und Assistenzärzten und der FMH. Letztere erhält so eine Antwort auf die Frage nach der Prozessqualität der Weiterbildung. Die Weiterbildungsstätten erhalten Hinweise auf mögliche Effizienzsteigerung der Weiterbildung. Die Assistenzärztinnen und Assistenzärzte erreichen eine Steigerung der Qualität und Effizienz der Weiterbildung. Durch ihre - subjektive - Beurteilung der Weiterbildungsstätten ermöglichen sie ein
Benchmarking zwischen ähnlichen Institutionen. Ein ungenügendes Resultat wird den Fachgesellschaften mitgeteilt und kann eine Visitation auslösen. Weiterbildungsstätten, die sich nicht an der Umfrage beteiligen, können die Anerkennung als solche durch die FMH verlieren und können in der Folge Assistenzärztinnen und Assistenzärzten keine anrechenbare Weiterbildung anbieten.

Wir hoffen auf eine gute Aufnahme des neuen Fragebogens durch alle Beteiligten und eine hohe Rücklaufquote.

\title{
Nouveau questionnaire d'évaluation des établissements de formation postgraduée - qu'en est-il?
}

\author{
Dr Max Giger, domaine de la formation médicale de la FMH \\ Michael Siegrist, p.d., Dr en phil., Centre de recherche sociale de l'Université de Zurich
}

Depuis six ans, une enquête est effectuée au début de l'automne auprès des médecins assistants sur la qualité des établissements de formation postgraduée. Les réponses reçues permettent, certes, une appréciation globale de ces établissements, sans toutefois renseigner sur les causes des lacunes relevées et les mesures ciblées qui permettraient de les combler. Dans deux cas, les résultats de l'enquête ont donné lieu à une visite des établissements concernés par la société de discipline. Dans les deux cas, le processus de formation postgraduée a pu être amélioré.

\section{Thèmes}

Un nouveau questionnaire a été créé dans le but de donner aux formateurs des établissements des indications plus concrètes sur la manière d'améliorer le processus de formation postgraduée. Le comité central de la FMH a chargé le centre de recherche sociale de l'Université de Zurich d'élaborer un nouveau questionnaire et de mener une enquête. Les thèmes ont été élaborés en deux ateliers, l'un avec les responsables des établissements de formation et l'autre avec des représentants des médecins assistants et chefs de clinique. Tous les protagonistes ont donc pu exercer leur influence sur le choix des critères destinés à mesurer la qualité de la formation postgraduée. Une publication et un débat ont suivi sur le site internet de la FMH. Une vingtaine de responsables d'établissements de formation postgraduée ont apporté leurs suggestions et émis leurs critiques, en partie après avoir débattu des thèmes traités avec leurs collaborateurs. Dans la mesure du possible, ces suggestions et critiques ont été prises en compte. Le questionnaire a ensuite été testé auprès de 12 médecins assistants des cliniques universitaires zurichoises et auprès de 15 médecins assistants des cliniques universitaires de Genève. Le nouveau questionnaire a été apprécié de manière positive et son envergure jugée acceptable. Les médecins assistants ont eu besoin de 10 à 15 minutes pour le remplir. L'expérience rassemblée au cours de ce test préliminaire, ainsi que les critiques émises, ont été prises en compte dans son remaniement. Le nouveau questionnaire sera envoyé dans la deuxième partie du mois d'août aux responsables des établissements de formation postgraduée pour les distribuer aux assistants. 


\section{Mise en œuvre et anonymat}

Le nouveau questionnaire s'appuie, sur le plan formel, sur le précédent. Il est quelque peu plus détaillé, mais nécessite moins d'un quart d'heure pour le remplir. Il est divisé en questions concernant la culture de la formation postgraduée, son exécution, la personne du médecin assistant et les études. Ces dernières questions n'ont été posées que cette année-ci. Elles sont notamment destinées à renseigner sur l'importance de la pratique dans les études. Les questions concernant la personne servent à interpréter les réponses aux autres questions. Toutes les données rassemblées demeurent auprès du bureau de recherche sociale. Tant la FMH que les responsables des établissements de formation reçoivent une évaluation des données anonymisées et résumées.

En présence d'au moins quatre questionnaires remplis auprès d'un établissement de formation, les responsables des établissements en question reçoivent un retour d'information contenant les valeurs moyennes de l'évaluation. S'il y a moins de quatre questionnaires, l'anonymat n'est plus vraiment garanti. Dès lors, les médecins assistants peuvent décider de les envoyer ou non. L'envoi de moins de quatre réactions aux responsables des établissements de formation est ainsi rendu possible.

\section{Conséquences de l'enquête}

Les résultats de cette enquête sont utiles aussi bien aux établissements de formation et aux médecins assistants qu'à la FMH. Cette dernière reçoit donc une réponse à la question de la qualité des processus de formation postgraduée. Les établissements, quant à eux, reçoivent des indications sur les possibilités d'augmenter l'efficacité de la formation postgraduée et les médecins assistants peuvent en augmenter la qualité et l'efficacité. Leur appréciation - subjective - des établissements de formation permet une analyse comparative entre des institutions similaires. Les résultats insuffisants, pouvant provoquer une visite de l'établissement en question, sont communiqués aux sociétés de discipline médicale. Les établissements de formation qui ne participent pas à l'enquête peuvent donc perdre leur reconnaissance par la $\mathrm{FMH}$, ce qui a pour conséquence, pour les médecins assistants, de voir leurs stages de formation postgraduée non reconnus.

Nous espérons que tous les participants feront bon accueil au nouveau questionnaire et qu'ils le renverront en grand nombre. 